\title{
Evidências internacionais de sonegação fiscal: uma análise a partir do modelo de Graetz, Reinganun e Wilde
}

\section{Felippe Clemente}

Pós-Doutorando em Economia - Universidade Federal de Viçosa (UFV)

Endereço: Av. PH Rolfs, s/n - Campus Universitário - Viçosa - Minas Gerais

CEP: 36590-000 - E-mail: felippe.clemente@ufv.br

\section{Viviani Silva Lírio}

Professora - Universidade Federal de Viçosa (UFV)

Endereço: Av. PH Rolfs, s/n - Campus Universitário - Viçosa - Minas Gerais

CEP: 36590-000 - E-mail: vslirio@ufv.br

Recebido: 19/06/2016. Aceite: 25/05/2017.

\section{Resumo}

O propósito desse artigo é parametrizar o modelo seminal de Graetz, Reinganun e Wilde (1986) para dois grupos de países: G1 com baixa evasão fiscal e G2 com alta evasão fiscal. Os principais resultados mostram uma forte correlação entre a carga tributária, o custo de fiscalizar e a evasão fiscal dos países. A multa parece ter pouca eficácia na mitigação da sonegação. Os resultados possuem implicações tanto do ponto de vista teórico quanto prático. Este é o primeiro estudo que se parametriza o modelo de Graetz, Reinganun e Wilde como forma de explanar a diversidade da evasão fiscal internacional e desenvolver uma estrutura internacional de conformidade fiscal. Assim, políticas que revisem a estrutura fiscal dos países bem como modernizem os órgãos fiscalizadores de forma a reduzir os seus custos, poderão ter efeitos positivos sobre a evasão de impostos.

\section{Palavras-Chave}

Sonegação fiscal. Teoria dos Jogos. Evidências internacionais.

\begin{abstract}
We propose to parameterize Graetz, Reinganun and Wilde's seminal model (1986) for two countries groups: G1 with low tax evasion and G2 with high tax evasion. Based on data from 16 countries, we find a strong correlation between tax burden, inspecting cost and countries' tax evasion. Fine seems to have lack of effectiveness in mitigation of evasion. Our results have implications for both research and practice. This is the first study to parametrize Graetz, Reinganun and Wilde's model as an explanator of international tax evasion diversity and serves as the starting point for the development of an international tax compliance framework. Thus, policies that review the countries' tax structure and modernize regulatory agencies to reduce their costs may have positive effects on tax evasion.
\end{abstract}

\section{Keywords}

Tax Evasion. Game Theory. International Evidences.

\section{JEL Classification}

D02. H26. H40.

- Os autores agradecem à Coordenação de Aperfeiçoamento de Pessoal de Nível Superior (CAPES) e ao Conselho Nacional de Desenvolvimento Científico e Tecnológico (CNPq) pelo financiamento das bolsas de estudos que possibilitaram a realização dessa pesquisa. 


\section{Introdução}

A sonegação fiscal é comum e recorrente nas mais diversas nacionalidades. Em países como Alemanha (36,6\%), ${ }^{1}$ Inglaterra $(32,1 \%)$ e Noruega $(38,7 \%)$, os quais possuem uma carga tributária alta, os agentes são estimulados a recorrer às práticas ilegais como forma de se manter no mercado. Já em países em desenvolvimento como México $(15,2 \%)$ e Chile $(19,8 \%)$, em que as cargas tributárias são baixas, os agentes sonegam com o objetivo de auferir maiores ganhos. Nesse sentido, a literatura traz diversos estudos que procuram analisar a complexidade da evasão e as variáveis que afetam a forma como os contribuintes sonegam (Clemente et al. 2014).

Allingham e Sandmo (1972) foram dois dos primeiros autores a estudar a evasão fiscal e se basearam no modelo seminal de Becker (1968), que estabeleceu a metodologia para a denominada Economia do Crime. De forma geral, Becker (1968) considerou as atividades criminais ${ }^{2}$ como decisão racional e individual que depende das probabilidades de detecção e convicção bem como do nível da punição.

Utilizando esse modelo, Allingham e Sandmo (1972) derivaram as condições sobre as quais o aumento nas penalidades ou na probabilidade de imposição das sanções aumentaria o montante declarado pelos contribuintes racionais. ${ }^{3}$ Embora a contribuição metodológica tenha sido significativa, os resultados não se mostraram particularmente úteis para a análise de políticas ou estudos empíricos, uma vez que os modelos consideraram apenas as ações dos contribuintes e ignoraram os agentes envolvidos no processo de coleta dos tributos (Siqueira 2004). ${ }^{4}$ Após a publicação de Allingham e Sandmo (1972), diversos pesquisadores despenderam esforços significativos para tentar compreender as dimensões que afetam e impactam a evasão fiscal.

1 Os dados entre parêntesis referem-se às cargas tributárias dos países para o ano de 2014. Esses dados são da OCDE e estão disponibilizados em http://www.oecd.org/tax/tax-policy/table-3-2-total-taxrevenue--gdp-1965-latest-year.htm.

2 Vale a pena destacar que a prática da evasão fiscal é considerada ilegal e, portanto, criminalizada na maioria dos países.

3 Considera-se como racional o contribuinte que maximiza sua renda dada às imposições e sanções dos órgãos fiscalizadores.

4 A seção 2.1.3 traz diversos trabalhos com extensões do modelo básico de Allingham e Sandmo (1972) com a finalidade de corrigir alguns entraves do modelo inicial. 
Pencavel (1978) analisou o modelo existente na literatura tradicional acerca da evasão fiscal que possui duas importantes implicações: i) o rendimento declarado às autoridades fiscais aumenta à medida que a carga tributária aumenta; e ii) dada uma carga tributária fixa, a renda verdadeiramente declarada cai com aumentos na renda nominal do contribuinte. Para isso, o autor realizou três modificações no modelo padrão. A primeira relaxa o pressuposto de carga tributária linear progressiva, buscando evidenciar o quanto as estruturas fiscais existentes se afastam dessa linearidade. A segunda modificação considera formas alternativas para as penalidades aplicadas ao sonegador de impostos, considerando violações graves com punições severas e violações menores em que o contribuinte paga apenas uma multa sobre os impostos evadidos. A última modificação proposta pelo autor conecta a variável declaração fiscal à oferta de mão de obra no mercado de trabalho. Aqui, o contribuinte é retratado como um tomador de decisão "ex-ante", que considera quantas horas trabalho ofertar e quanto irá declarar ao fisco as horas trabalhadas. A subdeclaração de impostos às autoridades afeta, por sua vez, o modelo convencional de oferta de mão de obra. Por meio do modelo proposto, o artigo mostrou que as implicações da literatura tradicional de evasão fiscal - carga tributária mais alta induz mais declarações ao fisco e o aumento da renda bruta induz em mais violações fiscais - possuem premissas fracas. As premissas são frágeis não porque entram em conflito com o comportamento observado do contribuinte, mas no sentido de terem sido construídas sob postulados muito estreitos. $\mathrm{O}$ autor conclui mostrando que a literatura requer trabalhos empíricos que confrontem as hipóteses e as ambiguidades derivadas em seu trabalho.

Clotfelter (1983) investigou a relação entre carga tributária marginal e a evasão fiscal com enfoque para os retornos dos indivíduos. Utilizando os dados do Serviço Interno de Receita Tributária dos EUA, o autor encontrou importantes resultados que mostram que a carga tributária marginal tem um efeito significativo sobre o montante evadido. A principal contribuição do trabalho foi prover evidências econométricas, sugerindo que a sonegação de impostos é sensível à carga tributária marginal. Com isso, faz-se necessário que as autoridades tributárias considerem a carga tributária juntamente com as variáveis "punição, auditoria e fiscalização" como instrumentos válidos que influenciam evasão fiscal.

Greenberg (1984) analisou a evasão fiscal do ponto de vista das autoridades tributárias e, para isso, utilizou jogos repetidos por serem modelos menos restritivos para se encontrar uma estratégia ótima. Para dinamizar a 
análise, o autor considerou três parâmetros que definem o jogo entre autoridade tributária e contribuintes: função de imposto $(\mathrm{t})$, função de penalidade (f) e a probabilidade de ser auditado (r). Entre os jogadores, esse modelo de interação é desenvolvido de forma a verificar os movimentos dos contribuintes entre diferentes grupos e, após as movimentações, encontrar o equilíbrio do jogo. Dentre os principais resultados, o autor destaca que os contribuintes consideram o tempo de vida e a perspectiva futura para decidirem evadir ou não. Entretanto, como os contribuintes não sabem, ao certo, a sua duração, a estratégia de agir honestamente deve funcionar ao menos com as gerações mais novas. Apesar da grande contribuição para a literatura, o autor aponta que uma suposição restritiva do modelo é que se o contribuinte é auditado, sua renda atual será, certamente, descoberta e haverá a multa certa a ser paga. Isso não condiz exatamente com a realidade, uma vez que há dificuldades judiciais em mensurar saber a renda verdadeira do contribuinte, bem como há vários tipos de multas a serem aplicadas.

Graetz e Wilde (1985) trazem três importantes recomendações para analisar, de forma mais concisa, a economia da conformidade fiscal (tax compliance). A primeira evidência dos autores versa sobre os dados empíricos e os erros cometidos pelos pesquisadores ao mensurar o montante sonegado. De fato, muitos economistas ignoram componentes importantes para se mensurar a sonegação fiscal, e não consideram variáveis legais, políticas e culturais. A segunda evidência enfatiza a limitação do modelo da Economia do Crime de Becker para explicar a sonegação fiscal. Os autores mostram que a sonegação fiscal depende muito da análise da interação entre o agente sonegador e as autoridades envolvidas no processo de fiscalização, além de ser necessário considerar a probabilidade de detecção de fraudes e evasão. Neste sentido, o modelo de Becker (1968) se mostra simplista demais para ser usado no ambiente complexo da tax compliance. Por fim, a terceira evidência é a recomendação dos autores aos formadores de políticas fiscais quando propõem acabar com a evasão de impostos por meio de aumentos na taxação sobre o consumo e diminuições na taxação da renda. De acordo com os autores, essa troca de base tributária não garante queda da sonegação, mas sim queda nas informações das declarações, o que se torna problemático na detecção da sonegação fiscal. A principal conclusão dos autores é que se faz necessário um refinamento da teoria acerca do tax compliance, além de estudos empíricos que desenhem melhores mecanismos de fiscalização e aprimore os que já existem. 
A partir desses estudos, ocorreu um avanço significativo efetuado por Graetz, Reinganun e Wilde (1986), que iniciaram a modelagem da dinâmica da evasão por meio de jogos de sonegação fiscal. O modelo passou a envolver os contribuintes, o fisco e os parâmetros que podem influenciar nas decisões dos agentes, permitindo, assim, análises empíricas e voltadas para analisar a eficiência das políticas. Entretanto, poucos estudos empíricos (ver Andreoni et al. 1998; Lipatov 2005, 2006) são observados na literatura, pois limitam-se apenas ao desenvolvimento no campo teórico. Isso pode mostrar a necessidade de estudos que verifiquem a robustez dos parâmetros nos estudos empíricos.

Alguns trabalhos recentes buscaram ampliar o modelo seminal de Graetz, Reinganun e Wilde - GRW (1986) além de aplicá-lo empiricamente. Andreoni et al. (1998) evidenciaram, empiricamente, as interações entre os contribuintes e os órgãos de fiscalização dos Estados Unidos, além de alguns parâmetros importantes que se relacionaram com os agentes tais como penalidades e custos. Os autores destacaram que há a necessidade de mais trabalhos empíricos e pesquisas institucionais fora dos Estados Unidos, haja visto que esse problema é recorrente em muitos outros países.

Lipatov (2008) estendeu o modelo GRW considerando as interações sociais e suas influências na evasão de impostos. Após a parametrização do modelo e a verificação da robustez dos parâmetros, o autor apresentou evidências importantes, mostrando o modelo que explica como o governo pode aumentar seu grau de confiança perante a sociedade e, com isso, reduzir a sonegação. Além disso, mostrou como ele pode prover, de forma ótima, os bens públicos, mitigando, assim, a evasão de impostos.

Assim, o que se pode observar na literatura é que os estudos sobre sonegação fiscal, de maior ou menor complexidade, versam sempre no sentido de explicar e de entender parâmetros importantes como multa sobre o ato sonegador, a carga tributária envolvida e o custo de fiscalizar e auditar os agentes. Além disso, há escassez de trabalhos empíricos (Andreoni et al. 1998).

Portanto, neste artigo, o objetivo é preencher, em parte, a lacuna existente na literatura, analisando empiricamente os parâmetros teóricos propostos em modelos de teoria dos jogos. O modelo escolhido para análise foi o de Graetz, Reinganun e Wilde - GRW (1986) por considerar interações mais realísticas entre contribuintes e autoridades tributárias. Os parâmetros 
analisados neste estudo compõem o modelo GRW e são os mais citados nos estudos científicos que englobam a sonegação fiscal: $\boldsymbol{s}$, multas; $\boldsymbol{t}$, carga tributária; $\boldsymbol{q}$, probabilidade de sonegação e $\boldsymbol{c}$, custo de auditoria.

Foram selecionados, para este estudo, dois grupos de países que se diferenciam na taxa de sonegação fiscal. ${ }^{5}$ O grupo 1 , composto por Alemanha, Canadá, Dinamarca, Noruega, Suécia, França e Holanda, é formado por países considerados com baixa evasão fiscal. O grupo 2, com Argentina, Bolívia, Colômbia, Chile, México, Peru e Brasil, países com alta taxa de evasão fiscal. A divisão dos países em dois grupos extremos ocorreu a fim de verificar suas características com relação à evasão fiscal de forma que o grupo 1 possa contribuir para com o grupo 2 .

O presente artigo está dividido em quarto seções. Além dessa introdução, o referencial teórico é apresentado seguido da metodologia e fonte dos dados, bem como dos resultados, discussões e considerações finais.

\section{Referencial teórico}

O trabalho de Graetz, Reinganun e Wilde (1986) contribuiu significativamente com a literatura, pois eles iniciaram os estudos de interação entre agentes sonegadores e órgãos de fiscalização. Nessa seção, há a exposição dos principais aspectos do modelo, bem como as considerações dos autores para os parâmetros utilizados. ${ }^{6}$

Suponha que alguns contribuintes são compliers habituais e, neste caso, eles reportam, verdadeiramente, sua renda, dados os seus interesses pecuniários. Os contribuintes agem estrategicamente, examinando seus incentivos, cuidadosamente, e agem de forma a maximizar a utilidade esperada, dada a probabilidade de fiscalização associada à renda que eles escolhem reportar.

Há, então, duas classes de renda, alta e baixa, denotadas por $\boldsymbol{H}$ e $\boldsymbol{L}$, respectivamente, onde, $\boldsymbol{L}<\boldsymbol{H}$ e $\boldsymbol{L}>\mathbf{0} ; \boldsymbol{H}>\mathbf{0}$. A renda dos contribuintes não é diretamente observável, de forma que eles podem reportar alta ou baixa

5 Vale ressaltar que há outras formas de classificar os grupos de países como instrumentos de fiscalização ou eficácia das sanções. Para esse estudo, resolveu-se selecionar os países de acordo com a taxa de sonegação fiscal.

6 Mais informações, consultar Graetz, Reinganun e Wilde (1986), seção 3 "A simple interactive model”. 
renda. $H$ representa a renda real e $L$ representa a renda reportada de forma que $\boldsymbol{H}$ - $\boldsymbol{L}$ representa a renda não reportada, ou seja, a parcela de renda que o agente pratica a evasão fiscal. Denota-se por $t$ o imposto individual a ser pago ao fisco. Assume-se que $\boldsymbol{t} \leq \boldsymbol{L}$ e $\boldsymbol{t} \leq \boldsymbol{H}$ e $\boldsymbol{t} \geq \mathbf{0}$. Ao contribuinte que for descoberto sub-reportando sua renda será aplicada uma multa ao montante sonegado denominada por $\boldsymbol{s},(\boldsymbol{s} \geq 0)$. Os parâmetros $\boldsymbol{t}$ e $\boldsymbol{s}$ são definidos como alíquota do imposto e percentual de multa, respectivamente.

Denota-se como custo de fiscalizar e auditar por $c$, em que $c \geq 0$. Para o modelo, supõe-se que $\boldsymbol{H}-\boldsymbol{L}+\boldsymbol{s}>\boldsymbol{c}$, ou seja, o incremento na receita do fisco associado às perdas com as sub-declarações excederam o custo de auditoria. Se isso não é verdade, então sempre que o agente sonegador puder ser identificado a priori, não seria vantajoso auditá-lo e, então, coletar impostos e multas, beneficiando-se, assim, este agente. Também se assume que $\boldsymbol{t}+\boldsymbol{s} \leq \boldsymbol{L}$ e $\boldsymbol{t}+\boldsymbol{s} \leq \boldsymbol{H}$ que é a soma das alíquotas dos impostos mais os percentuais de multas, não podem exceder a renda do contribuinte. Os autores também assumiram que os contribuintes que reportavam verdadeiramente nunca serão multados e não sofrerão com outros custos de auditoria.

Finalmente, $\boldsymbol{q}$ representa a probabilidade de escolha por parte do contribuinte de sonegar, onde $0<\boldsymbol{q}<1$. Da mesma forma, $\boldsymbol{p}$ representa a probabilidade de auditar por parte dos órgãos fiscalizadores, sendo $0<\boldsymbol{p}<1$. A partir dessas informações, é possível obter a função de utilidade do contribuinte e a receita esperada dos órgãos fiscalizadores $\pi(p, q)$, a saber:

$$
\begin{aligned}
& U(p, q)=p q(H-s t(H-L)-t(H-L)-1)+q t H+(1-t) H \\
& \pi(p, q)=p q t(H-L)(1+s)-q t(H-L)+t H-p c
\end{aligned}
$$

Essas informações estão disponíveis na forma de um jogo simultâneo no Quadro 1.

Quadro l - Jogo simultâneo entre contribuinte e o fisco

\begin{tabular}{|c|c|c|}
\hline & Auditar (p) & Não Auditar (1 - p) \\
\hline Evadir (q) & $(1-t) H-s t(H-L) ; t H+s t(H-L)-c$ & $H-t L ; t L$ \\
\hline Não Evadir (1 - q) & $(1-t) H ; t H-c$ & $(1-t) H ; t H$ \\
\hline
\end{tabular}

Fonte: Adaptado de Lipatov (2008) com base em Graetz, Reinganun e Wilde (1986). 
Nesse jogo, o contribuinte decide evadir ou não evadir impostos ao mesmo tempo em que a autoridade tributária decide auditar ou não auditar. As respectivas probabilidades de evasão e auditoria são, respectivamente, $q$ e $p$. Para cada ação do contribuinte e da autoridade tributária tem-se um payoff, que indica o ganho de cada agente de acordo com a sua ação. Por exemplo, no caso de o contribuinte evadir impostos e a autoridade fiscalizá-lo, implementa-se o percentual de multa $(s)$ sobre o montante sonegado e o custo de auditoria $(c)$.

\section{Procedimentos metodológicos e fonte dos dados}

A partir do modelo de Graetz, Reinganun e Wilde (1986) descrito, foram calculados os payoff dos órgãos fiscalizadores no equilíbrio de estratégias mistas para jogos simultâneos, com a finalidade de encontrar a relação entre o parâmetro $c$ e os demais parâmetros do modelo. O equilíbrio em estratégias mistas considera as probabilidades de o agente sonegar ou não, dado que os órgãos fiscalizados realizem a auditoria ou não. Assim, tem-se:

$$
\begin{aligned}
& \pi_{1}^{e}=q[(t H+s t(H-L)-c]+(1-q)[t H-c] \\
& \pi_{2}^{e}=q(t L)+(1-q)[t H] \\
& \pi_{1}^{e}=\pi_{2}^{e} \\
& q[(t H+s t(H-L)-c]+(1-q)[t H-c]=q(t L)+(1-q)[t H] \\
& \boldsymbol{c}=\boldsymbol{q} \boldsymbol{t}(\boldsymbol{H}-\boldsymbol{L})(\mathbf{1}+\boldsymbol{s})^{8}
\end{aligned}
$$

Os valores $\pi_{1}^{e}$ e $\pi_{2}^{e}$ indicam os payoff do fisco quando ele audita e não audita, respectivamente. No equilíbrio, é considerada a igualdade entre os payoff $\left(\pi_{1}^{e}=\pi_{2}^{e}\right)$ e, após esse procedimento, tem-se o parâmetro $c$ em relação às demais variáveis.

Os parâmetros do modelo GRW possuem os seguintes significados: $\boldsymbol{s}$, percentual de multa, é a penalidade imposta pelas autoridades fiscais para o montante não declarado; $t$, alíquota de imposto, é o montante total sobre

8 A derivação completa do modelo encontra-se no anexo. 
a renda bruta no qual o contribuinte precisa repassar ao fisco; $\boldsymbol{H}-\boldsymbol{L}$, sonegação fiscal, é a diferença entre o valor real $H$ e o valor declarado $L$ para os órgãos de fiscalização; $\boldsymbol{q}$, probabilidade de evasão fiscal, envolve as chances do contribuinte em sonegar impostos e $c$, custo de auditoria, é o custo que as autoridades tributárias possuem ao iniciar a fiscalização do contribuinte.

Utilizou-se da estatística descritiva média e das medidas de dispersão desvio-padrão, curtose e coeficiente de assimetria, com a finalidade de comparar os parâmetros $\boldsymbol{s}, \boldsymbol{t}, \boldsymbol{q}$ e $\boldsymbol{c}$ entre os grupos bem como para facilitar a análise dos dados coletados. Esses são calculados da seguinte forma: i) média; ii) desvio-padrão que mede a dispersão dos valores em torno da média; iii) curtose que mede o grau de achatamento da distribuição dos dados ${ }^{9}$ e iv) coeficiente de assimetria que resulta em um grau de afastamento da simetria de uma distribuição de dados. ${ }^{10}$

Com relação aos países estudados, foram considerados 14 países divididos em dois grupos: o primeiro grupo de países com baixa evasão e o segundo grupo composto por países com alta evasão fiscal. Para a escolha dos países dois estudos foram utilizados. Buehn e Schneider (2012) calcularam a taxa de sonegação fiscal (\% do PIB) para os países da Organização para Cooperação e Desenvolvimento Econômico (OECD) para o ano de 2010. A partir daí os sete países foram escolhidos por apresentarem as menores taxas de evasão, a saber: Alemanha, Canadá, Dinamarca, Noruega, Suécia, França e Holanda. O outro estudo utilizado foi de Jiménez e Sabaini (2012), que calcularam a taxa de sonegação fiscal para os países da América Latina para 2010. Os autores ressaltaram que a evasão de impostos na América Latina é um dos maiores do mundo, o que nos permite considerá-la no grupo dois. Com isso, foram considerados os sete países com maiores taxas de sonegação, a saber: Argentina, Bolívia, Colômbia, Chile, México, Peru e Brasil.

\footnotetext{
9 Nesse caso, se o resultado for zero, a distribuição de frequências será a própria distribuição normal denominada mesocúrtica. Se for negativo, a distribuição será achatada, indicando alta variabilidade dos dados e chamada de planicúrtica e, se for positivo, ela será concentrada em torno da média, evidenciando alta homogeneidade e chamada de leptocúrtica.

${ }^{10}$ No caso de uma assimetria negativa, inclinada para a esquerda, existirão mais valores da amostra maiores que a média. Em uma assimetria positiva, existirão mais valores da amostra menores que a média, tendo a curva da distribuição uma cauda mais longa em relação aos valores maiores que a média, cujo valor é maior que a mediana que é maior que o da moda.
} 
Para os parâmetros do modelo, as fontes dos dados foram diversas. Para o parâmetro da multa pelo montante evadido $(s)$ pesquisaram-se informações dos valores nas plataformas on-line dos países tais como Receitas Federais e Ministérios Públicos. Para os países da Europa, a plataforma European Tax Justice ${ }^{11}$ fornece detalhadamente como funcionam as penalidades e as sanções impostas pelos países no combate à sonegação.

O parâmetro da taxa de evasão fiscal realizada ( $\mathrm{H}$ - L) foi extraído dos estudos de Buehn e Schneider (2012) e Jiménez e Sabaini (2012). Para os parâmetros $\boldsymbol{t}$ e $\boldsymbol{q}$, carga tributária e probabilidade de sonegação fiscal, respectivamente, as informações foram extraídas do Global Competitiveness Report de 2010 o qual contém dados econômicos e institucionais dos países analisados. Como o parâmetro $\boldsymbol{q}$ envolve probabilidade, ele foi construído da seguinte forma: considerou-se a variável efficiency of tax regulations, também extraída do Global Competitiveness Report de 2010, que fornece o score de pontos para cada país. A partir disso, calculou-se a regra dos três termos inversa, indicando os países com altos scores como tendo baixas probabilidades de sonegação fiscal e países com baixos scores com altas probabilidades. Assim, a França, por exemplo, que possui score de 14,5, tem probabilidade de $11,60 \%$ de sonegação fiscal. Já o Uruguai, com score de 2,5 , tem probabilidade de $66,2 \%$ de sonegação fiscal. O parâmetro $c$, custo de auditar, foi calculado a partir da Equação (5).

As Tabelas 1 e 2 trazem os valores dos parâmetros do modelo de GRW utilizados no estudo. A Tabela 1 refere-se aos países do Grupo l e a Tabela 2 aos países do Grupo 2.

Tabela 1 - Valores dos parâmetros para os países do Gl

\begin{tabular}{ccccc}
\hline Países & Multa $(\mathbf{s})$ & Evasão $(\mathbf{H}-\mathbf{T})$ & Probabilidade (q) & Imposto $(\mathbf{t})$ \\
\hline Alemanha & 0.15 & 1.1 & 0.100 & 0.488 \\
Canadá & 2 & 0.8 & 0.198 & 0.21 \\
Dinamarca & 0.75 & 1.7 & 0.111 & 0.26 \\
Finlândia & 0.3 & 1.5 & 0.131 & 0.4 \\
Noruega & 0.3 & 1.7 & 0.267 & 0.407 \\
Suécia & 0.4 & 1.7 & 0.173 & 0.494 \\
França & 0.8 & 1.1 & 0.116 & 0.666 \\
Holanda & 2 & 1.3 & 0.132 & 0.39 \\
\hline
\end{tabular}

Fonte: Diversos.

${ }^{11}$ Mais informações: http://www.taxjustice.net/tag/eu/. 
Tabela 2 - Valores dos parâmetros para os países do G2

\begin{tabular}{ccccc} 
Países & Multa $(\mathbf{s})$ & Evasão $(\mathbf{H}-\mathbf{T})$ & Probabilidade $(\mathbf{q})$ & Imposto $(\mathbf{t})$ \\
\hline Argentina & 2 & 21.2 & 0.672 & 0.68 \\
Bolívia & 1 & 29 & 0.480 & 0.837 \\
Chile & 3 & 11 & 0.213 & 0.279 \\
Colômbia & 1 & 23.5 & 0.189 & 0.754 \\
México & 1 & 20 & 0.200 & 0.518 \\
Peru & 1 & 37.3 & 0.280 & 0.36 \\
Uruguai & 1.5 & 26.3 & 0.662 & 0.418 \\
Brasil & 1.5 & 27.6 & 0.177 & 0.69 \\
\hline
\end{tabular}

Fonte: Diversos.

Destaca-se, conforme Jiménez e Sabaini (2012), o aumento excessivo da carga tributária na América Latina que vem ocorrendo desde os anos 1990. Devido à falta de estrutura nas fiscalizações e no combate à evasão fiscal, os países têm encontrado, no aumento dos tributos, formas de aumento das receitas fiscais, ao invés de se investir em mecanismos de controle da sonegação de impostos. Exemplos extremos podem ser observados para a Bolívia (83.7\%) e para a Colômbia (75.4\%) que possuem cargas tributárias altíssimas seguidos de altas taxas de sonegação fiscal, $29 \%$ e $23.5 \%$.

A partir daqui, iniciam-se algumas comparações gráficas dos parâmetros a fim de evidenciar os valores dos parâmetros entre os grupos de países. As variáveis utilizadas nessa parte do estudo são: o custo de fiscalização $(c)$, o percentual de multas aplicadas aos sonegadores $(\boldsymbol{s})$, a alíquota de impostos $(\boldsymbol{t})$, a probabilidade dos agentes evadirem $(\boldsymbol{q})$ e a evasão efetivamente concretizada $(\boldsymbol{H}-\boldsymbol{L})$. Note que as variáveis $\boldsymbol{q}$ e $\boldsymbol{H}-\boldsymbol{L}$ diferem no conceito e também nas grandezas, pois à medida que $\boldsymbol{q}$ pode variar entre 0 e 1 , o valor $H$ - $\boldsymbol{L}$ é a representação da evasão fiscal de cada país em termos monetários. Todas as variáveis, nesta etapa, foram analisadas de forma logarítmica a fim de aproximar as grandezas dos dados. ${ }^{12}$

O Gráfico 1 mostra a relação entre os parâmetros $t$, alíquota de impostos, e $\boldsymbol{H}-\boldsymbol{L}$, evasão fiscal efetiva. Observa-se que ambos os parâmetros oscilam de forma similar e na mesma direção. Assim, os países mais à esquerda no eixo das abscissas e que compõem o grupo 1 - países com baixa evasão fiscal - possuem também baixas alíquotas de impostos. Já os países mais à

12 Assim, por exemplo, a partir daqui, ao dizer, o parâmetro $\boldsymbol{t}$, subentende-se o logaritmo do parâmetro $\boldsymbol{t}$. 
direita, os países do grupo 2 - países com alta evasão fiscal - possuem também altas alíquotas. O que se pode, de fato, evidenciar, é que a alíquota de impostos e a evasão fiscal tendem a ter trajetórias com comportamento similar e, no mesmo sentido, nos leva a inferir que há uma alta correlação entre as variáveis.

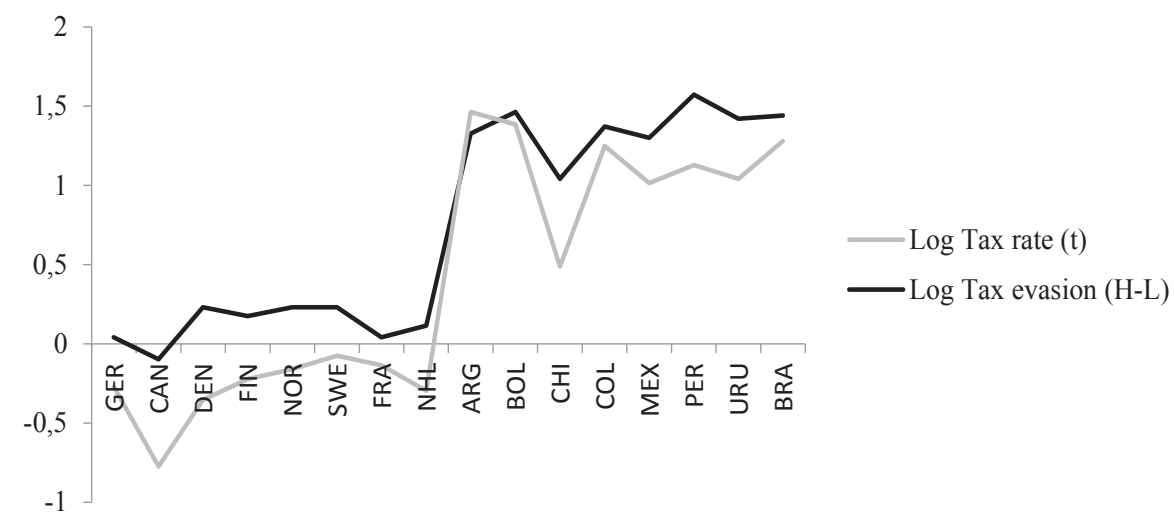

Gráfico 1 - Relação entre $t$ e $H-L$ dos países selecionados Fonte: dados da pesquisa.

Esse resultado corrobora com os encontrados em outras pesquisas científicas. Chiarini et al. (2013) fizeram uma breve survey da literatura, mostrando como os pesquisadores têm trabalhado sobre esse tema. Dentre esses, vale destacar o trabalho seminal de Allingham e Sandmo (1972), que identificou uma relação positiva entre alíquota de impostos e evasão fiscal que depende da aversão ao risco das penalidades existentes contra a sonegação. Outros pesquisadores ampliaram o modelo de Allingham e Sandmo com o objetivo de torná-lo mais robusto e evidenciaram que, para determinados valores de alíquotas e penalidades existentes, pode ser racional para os agentes sonegarem muito mais do que eles aparentam sonegar (Slemrod e Yitzhaki 2000). Em análises mais recentes e utilizando a Survey on Household Income and Wealth of Italy, Fiorio e Zanardi (2006) enfatizaram que a principal causa da evasão fiscal na Itália é a alta alíquota tributária imposta.

No que tange as análises para o Gráfico 1, observa-se, em suma, que tanto nesta seção quanto na literatura, a alíquota de impostos e evasão fiscal são altamente correlacionadas e positivas, sinalizando necessidades de mu- 
danças na estrutura do fisco como possível caminho para a redução da sonegação.

Analisando outras variáveis do modelo teórico proposto, o Gráfico 2 visa relacionar os parâmetros $\boldsymbol{s}$, percentual de multa aos agentes sonegadores e $\boldsymbol{H}-\boldsymbol{L}$, evasão fiscal efetiva.

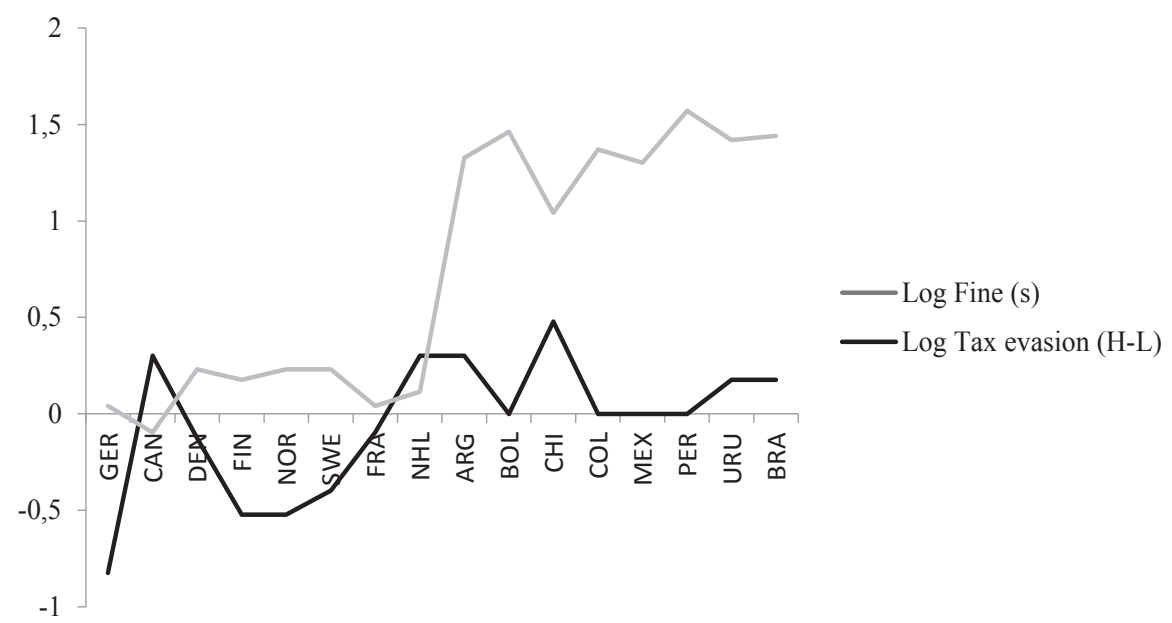

Gráfico 2 - Relação entre $s$ e $H$ - $L$ dos países selecionados

Fonte: dados da pesquisa.

Observa-se aqui que os parâmetros $\boldsymbol{s}$ e $\boldsymbol{H}-\boldsymbol{L}$ não oscilam conjuntamente e nem na mesma direção, tanto para o primeiro quanto para o segundo grupo de países. Mais uma vez pode-se auferir a baixa correlação entre os percentuais de multas aplicados pelos órgãos fiscalizadores e a sonegação fiscal. Mostrando possíveis interações entre agentes sonegadores, Lipatov (2006) evidenciou que o efeito dos percentuais de multas dependerá crucialmente do padrão contábil ${ }^{13}$ adotado pelos agentes. Se o padrão contábil escolhido for do tipo agressive, ${ }^{14}$ o aumento no percentual de multas pode ter efeito adverso na fiscalização. Em termos políticos, o autor sugere que, em países com altas taxas de evasões fiscais, o aumento dos percentuais de multas pode levar a resultados não esperados.

\footnotetext{
13 Padrão contábil: a estratégia que será utilizada para reportar os valores ao fisco.

14 Ocorre quando a interação entre os agentes é máxima, transparecendo ser um só agente ao fisco.
} 


\section{Resultados e discussão}

Os resultados do presente artigo objetivam avaliar os parâmetros $\boldsymbol{s}$, o percentual de multa sobre evasão, $\boldsymbol{q}$, a probabilidade de evasão fiscal pelo agente, $\boldsymbol{t}$, a alíquota de imposto sobre a renda bruta e $\boldsymbol{c}$, o custo de auditoria do modelo teórico proposto. Essa parametrização se torna um primeiro esforço no sentido de compreender a influência dessas variáveis na probabilidade de os agentes dos países sonegarem. Essas análises são importantes, pois permitem conhecer quais instrumentos eficientes essas esferas governamentais podem utilizar para mitigar a evasão fiscal. Nesse sentido, como apoio complementar às análises, fez-se, primeiramente, o cálculo da média $(\mu)$ e das medidas de dispersão desvio-padrão $(\sigma)$, curtose $(K)$ e coeficiente de assimetria (As), conforme pode ser observado na Tabela 3.

Iniciando com o parâmetro $\boldsymbol{s}$, foi observada uma média 1,169 (aproximadamente $116 \%$ ) com a curtose e o coeficiente de assimetria positivos. Isso implica a homogeneidade dos dados em torno da média, sendo, porém, a maior parte das observações menores que o valor médio. Apenas alguns países como Chile, Argentina, Holanda e Canadá apresentaram valores altos para os percentuais de multas sobre evasão fiscal na ordem de $300 \%{ }^{15}$ para o Chile e $200 \%$ para os demais países.

O parâmetro $\boldsymbol{q}$ apresenta a média 0,257 (em torno de $26 \%$ ) com valores positivos para a curtose e o coeficiente de assimetria. Isso indica que, em média, há a probabilidade de $26 \%$ dos agentes sonegadores dos países realizarem, de fato, a evasão fiscal. As medidas de dispersão mostram que os valores estão concentrados próximos ao valor médio, entretanto, com uma concentração dos dados à esquerda da distribuição.

Tabela 3 - Análise estatística agregada para os países selecionados.

\begin{tabular}{ccccc}
\hline & $\boldsymbol{\mu}$ & $\boldsymbol{\Sigma}$ & $\boldsymbol{K}$ & $\boldsymbol{A s}$ \\
\hline Multa (s) & 1,169 & 0,784 & 0,341 & 0,787 \\
Probabilidade (q) & 0,257 & 0,186 & 1,615 & 1,641 \\
Imposto (t) & 0,509 & 0,219 & $-0,171$ & 0,733 \\
Custo auditoria (c) & 7,137 & 11,604 & 4,875 & 2,183 \\
\hline
\end{tabular}

Fonte: dados da pesquisa.

${ }^{15}$ Esse valor indica que se cobra uma multa de 3 vezes sobre o montante sonegado. 
As maiores probabilidades de evasão se concentraram em países da América Latina, a saber: Argentina 67\%, Uruguai 66\%, Bolívia 48\% e Peru 28\%. Já os países com menores chances de sonegação foram Alemanha 10\%, Dinamarca 11\%, França 11,7\% e Finlândia 13\%. Vale lembrar que o parâmetro $\boldsymbol{q}$ foi calculado a partir da variável "eficiência das instituições de regulação fiscal" do Global Competitiveness Report que sinaliza a percepção dos agentes com relação às instituições.

Assim, para altos valores na eficiência das instituições, considerou-se baixa a probabilidade de sonegar. O contrário é válido para os baixos valores na eficiência reportados no relatório. No parâmetro $t$ tem-se média 0,534 (53,4\% de alíquota de imposto). As medidas de dispersão apresentam valores positivos, exceto a curtose, o que indica alta variabilidade dos dados em relação ao valor médio. Os valores extremos foram encontrados para os países Canadá 21\% e Bolívia 83,7\%, indicando a diferença de 62,7 p.p. nas alíquotas entre esses países.

Por fim, o parâmetro $c$, que apresenta o custo de auditoria em termos monetários, mostrou-se com média 7,137 e altos valores positivos nas medidas de dispersão, indicando grande quantidade de observações à esquerda da curva de distribuição. Os países com os menores custos de auditar e fiscalizar são: Alemanha 0,06, Dinamarca 0,08 e Canadá 0,09. Já os países com maiores custos são: Argentina 41,8, Bolívia 23,3, Uruguai 18,5 e Brasil 8,42. Isso significa dizer, por exemplo, que para cada unidade física adicionada na autoridade tributária da Alemanha, tem-se custo monetário de 0,06 e para cada unidade física inserida nos órgãos de fiscalização na Argentina tem-se um custo monetário de $41,8 .{ }^{16}$ A Tabela 4 evidencia os valores para o parâmetro $c$ para todos os países.

16 Ressalta-se aqui que o foco não está propriamente na representação monetária do parâmetro $c$, mas sim na grandeza do valor encontrado para cada país. Estudo mais aprofundado e detalhado seria necessário para analisar o custo de cada unidade incremental na fiscalização da sonegação fiscal dos países, o que fugiria ao escopo do presente artigo. 
Tabela 4 - Custo de Auditoria para os países selecionados

\begin{tabular}{cccc}
\hline Países & $\boldsymbol{c}$ & Países & $\boldsymbol{c}$ \\
\hline Alemanha & 0,06173 & Argentina & 41,8844 \\
Canadá & 0,09961 & Bolívia & 23,3021 \\
Dinamarca & 0,08549 & Chile & 2,61059 \\
Finlândia & 0,10238 & Colômbia & 6,68942 \\
Noruega & 0,23986 & México & 4,144 \\
Suécia & 0,20363 & Peru & 7,51968 \\
França & 0,15278 & Uruguai & 18,4689 \\
Nova Zelândia & 0,2012 & Brasil & 8,41945 \\
\hline
\end{tabular}

Fonte: Dados da pesquisa.

Sumarizando as informações da Tabela 3, pode-se inferir que países que possuem menores valores para o parâmetro $c$ indicam pequenas probabilidades de sonegação fiscal $(\boldsymbol{q})$. Para corroborar com esse resultado, calculou-se o coeficiente de correlação de Pearson e obteve-se $r=0,89,{ }^{17}$ indicando alta correlação entre o custo de fiscalizar e a probabilidade de evasão. Já os parâmetros $\boldsymbol{s}$ e $\boldsymbol{q}$ não se mostram altamente correlacionados $(r=0,29)$. Deve-se ressaltar que essa correlação possui valor fixo. Isto significa que os maiores sonegadores são menos penalizados, comparativamente. Como foi observada significativa variabilidade dos dados, optou-se por calcular e analisar as médias e as medidas de dispersão dos parâmetros por grupo. Assim, na Tabela 3, estão indicados os valores estatísticos para os grupos 1 e 2 .

Observando o parâmetro $s$ percebe-se que para o grupo 1 a média é menor que do grupo 2. Na Tabela 5, a curtose apresenta-se com valor negativo para o Gl, o que indica certa variabilidade dos dados ao longo da distribuição. O contrário ocorre no G2, evidenciando alta homogeneidade dos dados em torno da média. O que se pode inferir mais uma vez é a pouca eficácia dos percentuais de multas aplicados aos sonegadores, haja vista que $\boldsymbol{s}$ possui valor médio maior nos países com alta taxa de evasão de impostos.

${ }^{17}$ Coeficiente de correlação de Pearson: $r=\frac{\sum\left(x_{i}-\bar{x}\right)\left(y_{i}-\bar{y}\right)}{\sqrt{\left(\sum\left(\left(x_{i}-\bar{x}\right)^{2}\right)\left(\sum\left(y_{i}-\bar{y}\right)^{2}\right)\right.}}$. 
Tabela 5 - Análise estatística para os Grupos 1 e 2

\begin{tabular}{c|ccccccccc}
\hline & \multicolumn{3}{|c}{$\boldsymbol{\mu}$} & \multicolumn{2}{c}{$\sigma$} & \multicolumn{3}{c}{$\boldsymbol{K}$} & \multicolumn{2}{c}{$A s$} \\
\hline & G1 & G2 & G1 & G2 & G1 & G2 & G1 & G2 \\
\hline Multa (s) & 0,838 & 1,500 & 0,752 & 0,707 & $-0,516$ & 2,471 & 1,087 & 1,616 \\
Probabilidade (q) & 0,153 & 0,360 & 0,056 & 0,216 & 1,309 & $-1,373$ & 1,323 & 0,820 \\
Imposto (t) & 0,414 & 0,605 & 0,142 & 0,249 & 0,460 & $-1,363$ & 0,305 & 0,164 \\
Custo auditoria (c) & 0,143 & 14,130 & 0,065 & 13,295 & $-1,663$ & 2,061 & 0,288 & 1,526 \\
\hline
\end{tabular}

Fonte: dados da pesquisa.

O parâmetro $\boldsymbol{q}$ também mostra discrepância entre os valores apresentados na Tabela 5. Enquanto que no G2 a curtose se mostra negativa, no G1 ela é positiva, o que indica alta variabilidade e homogeneidade dos dados, respectivamente. Para os países do grupo 2, a probabilidade de evadir é, em média, de $36 \%$, ao passo que para os países do grupo 1, a probabilidade cai para $15 \%$. Percebe-se que há a significativa diferença (2l p.p.) entre os grupos quando se trata da possibilidade de sonegar ou não. Analisando o parâmetro $t$ que se mostra com valor médio de $41,4 \%$ para o grupo 1 e de $60,5 \%$ para o grupo 2 , observa-se alta alíquota de imposto para o grupo 2 (países com alta taxa de evasão), evidenciando mais a influência positiva da magnitude das alíquotas sobre a sonegação. $\mathrm{O}$ último parâmetro a ser analisado é o $c$, custo de fiscalizar. O que se observa são valores médios muito discrepantes entre os grupos 1 e 2, a saber, 0,143 e 14,13, respectivamente. Essa diferença corresponde a 13,987 , variação de $9.900 \%$ a mais que o custo de auditar nos países do grupo 1. Isso mostra um grande problema nos países com alta taxa de evasão, o que pode nos levar a inferir, em análise preliminar, que o custo da fiscalização está altamente correlacionado à probabilidade de sonegar tributos.

Resumidamente, o que se evidencia na Tabela 5 é a grande diferença dos parâmetros $\boldsymbol{s}, \boldsymbol{q}, \boldsymbol{t}$ e $\boldsymbol{c}$ entre os grupos dos países bem como a distribuição desses dados em torno da média. Além disso, os custos de auditoria e fiscalização são menores em países com baixa sonegação fiscal, ao contrário dos países da América Latina que possuem altas taxas de evasão de impostos. 
A análise do Gráfico 3 refere-se à interação $c$ e $\boldsymbol{q}$, custo de fiscalizar e probabilidade de evasão de impostos.

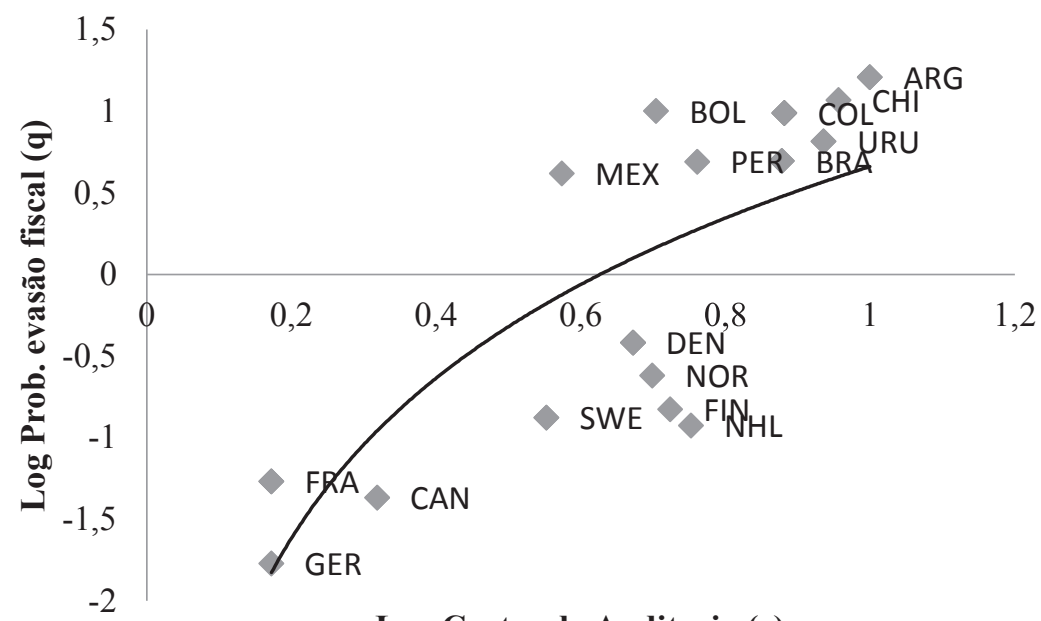

Log Custos de Auditoria (c)

Gráfico 3 - Interação entre $c$ e $q$ dos países selecionados

Fonte: dados da pesquisa.

A interação entre as variáveis mostra-se positiva para os países estudados neste trabalho, o que evidencia que aumentos incrementais nos custos de auditoria e fiscalização aumentam diretamente a probabilidade de evasão fiscal. Nesse sentido, alguns trabalhos corroboram com o resultado encontrado. Ao concluir as análises das modelagens, Lipatov (2006) evidenciou que os custos de auditoria podem ter o seguinte efeito: i) investimentos em formas alternativas de inspeção não traz resultados positivos para o fisco quando se trata de países em que a evasão é realmente alta. Faz-se necessária intensidade na auditoria dos agentes. Em países com sonegação moderada, a inspeção alternativa tornar-se-ia mais efetiva que multas ou auditorias intensas. Isso decorre do fato que países com pouca evasão já possuem fiscalizações, sendo, então, necessário apenas incrementar técnicas de inspeção.

Estudando o Brasil, Batista Júnior (2000) enfatizou que a complexidade do sistema tributário é o principal fator que dificulta a fiscalização dos agentes sonegadores. A falta de auditores para a realização do trabalho do 
fisco também se apresenta deficitário. No seu estudo, o autor comparou o número de auditores por habitantes do Brasil com países selecionados da OECD. A França possui 497 habitantes para cada fiscal; Alemanha 729 e o Canadá, 1.653. Vale lembrar que, além do expressivo número de fiscais, a administração tributária desses países está em patamar tecnológico elevado e na simplificação do fisco, como ocorreu recentemente nos países europeus com o imposto único. O Brasil contava, em 2000, com 12.000 auditores federais para a população de 195 milhões de habitantes. Isso resulta em um auditor para cada 4.646 habitantes, muito abaixo do padrão internacional. Além disso, o sistema brasileiro é muito complexo, o que dificulta a fiscalização por parte dos agentes.

Sumarizando as análises acima, de fato, como mostra esse estudo e estudos encontrados na literatura, o custo de auditoria impacta na probabilidade do agente de sonegar. Isso nos mostra que políticas que diminuam esse custo são bem vistas para mitigar o problema da evasão fiscal, ceteris paribus.

\section{Considerações finais}

O presente estudo encontrou resultados empíricos acerca dos principais parâmetros teóricos que se relacionam à evasão fiscal dos países. Os principais resultados a serem enfatizados são a expressiva correlação entre a alíquota de imposto $(\boldsymbol{t})$ e a evasão $(\boldsymbol{H}-\boldsymbol{L})$ e entre o custo de fiscalização $(\boldsymbol{c})$ e a decisão do agente de sonegar impostos $(\boldsymbol{q})$. Outro resultado importante é a baixa correlação do percentual de multa $(\boldsymbol{s})$ e a evasão de impostos. Isso pode trazer a discussão onde o poder público pode atuar de forma a mitigar efetivamente a sonegação. Medidas que diminuam o custo de fiscalizar e as alíquotas tributárias podem se mostrar mais eficazes que o aumento do percentual de multa aos sonegadores.

O artigo contribui com a literatura por ser um estudo empírico que utiliza dados de dois diferentes grupos de países para testar o modelo teórico de jogos, preenchendo, em parte, as lacunas existentes no campo prático para os modelos teóricos, conforme destacou Andreoni et al. (1998). O que se observa, de modo geral, na literatura são extensões ao modelo de Graetz, Reinganun e Wilde (1986), mas sempre no campo teórico e com poucas aplicações aos dados existentes. 
Uma das limitações desse estudo pode se constituir do fato de levar em consideração poucos países na análise e apenas de 2 (duas) regiões. Embora as informações sejam restritas, ampliar os grupos de países estudados pode fornecer informações de maior robustez para os parâmetros. Como sugestão para trabalhos futuros, recomenda-se utilizar as extensões aos modelos Lipatov (2006; 2008), juntamente com os dados disponíveis e realizar o estudo de forma empírica.

\section{Referências}

Andreoni, James, Brian Erard and Jonathan Feinstein. 1998. "Tax Compliance.” Journal of Economic Literature: 818-860.

Aguiar, Alexandre Street de. 2008. "Equivalente certo e medidas de risco em decisões de comercialização em energia elétrica.” Tese de doutorado, Pontifícia Universidade Católica (PUC-RIO), Rio de Janeiro.

Allingham, Michael G., and Agnar Sandmo. 1972. "Income Tax Evasion: A Theoretical Analysis.” Journal of Public Economics 1: 323-338.

Batista Jr, Paulo Nogueira. 2000. “Fiscalização tributária no Brasil: uma Perspectiva Macroeconômica.” Instituto de Estudos Avançados USP: 1-63.

Becker, Gary S. 1968. "Crime and Punishment: an Economic Approach.” Journal of Political Economy 76: 169-217.

Buehn, Andreas, and Friedrich Schneider. 2012. "Size and Development of Tax Evasion in 38 OECD Countries: What do we (not) know?” CESifo Working Paper n. 4004: 1-22.

Chiarini, Bruno, Elisabetta Marzano and Friedrich Schneider. 2013. "Tax rates and tax evasion: an empirical analysis of the long-run aspects in Italy.” European Journal of Law and Economics 35: 273-293.

Clotfelter, Charles. 1983. "Tax evasion and tax rates: an analysis of individual returns.” The Review of Economics and Statistics 62 (3): 363-373. MIT Press.

Fiorio, C.V., A. Zanardi. 2006. “L'evasione fiscal: Cosa ne pensano gli italiani?” La finanza publica italiana: 1-21. Graetz, M, and L. Wilde. 1985. "The economics of tax compliance: fact and fantasy.” National Tax Journal 38 (3): $355-363$.

Graetz, M, J. Reinganum and L. Wilde. 1986. "The Tax Compliance Game: Towards an Interactive Theory of Law Enforcement.” Journal of Law, Economics and Organization 2 (1): 1-32.

Greenberg, Joseph. 1984. “Avoiding tax avoidance: a (repeated) game-theoretic approach.” Journal of Economic Theory 31: 1-13. doi:10.1016/0022-0531(84)90071-1.

Lipatov, Vilen. 2005. “Corporate Tax Evasion: the Case for Specialists.” Munich Personal RePEc Archive (MPRA) 24472: 1-29. doi:10.1016/j.jebo.2011.09.015.

Lipatov, Vilen. 2006. “Compatibility in Tax Reporting.” Munich Personal RePEc Archive (MPRA) 21542: 1-29. Lipatov, Vilen. 2008. "Social Interaction in Tax Evasion.” Munich Personal RePEc Archive (MPRA) 8829: 1-35. Pencavel, John. 1979. "A note on income tax evasion, labor supply, and nonlinear tax schedules." Journal of Public Economics 12: 115-124. North-Holland Publishing Company. 
Sabaini, Juan Carlos Gómez, and Juan Pablo Jiménez. 2012. "Tax structure and tax evasion in Latin America." CEPAL Economic Development Division: 1-82.

Siqueira, Marcelo Lettieri. 2004. “Um modelo econômico para análise da evasão fiscal do imposto sobre a renda no Brasil.” Tese, Universidade Federal de Pernambuco, Recife. Programa de Pós-Graduação em Economia - PIMES. Slemrod, Joel, and Shlomo Yitzhaki. 2000. "Tax avoidance, evasion and administration.” NBER Working Paper n. 7473: 1-32.

\section{Anexo}

Derivação completa do Modelo Metodológico proposto

$$
\begin{gathered}
\pi_{2}^{e}=q(t L)+(1-q)[t H] \\
\pi_{1}^{e}=\pi_{2}^{e} \\
q[(t H+s t(H-L)-c]+(1-q)[t H-c]=q(t L)+(1-q)[t H] \\
q t H+q s t H-q s t L-q c+t H-c-q t H+q c=q t L+t H-q t H \\
q t H+q s t H-q s t L-c=q t L \\
c=q t H+q s t H-q s t L-q t L \\
c=q t[H+s H-s L-L] \\
\boldsymbol{c}=\boldsymbol{q} \boldsymbol{t}(\boldsymbol{H}-\boldsymbol{L})(\mathbf{1}+\boldsymbol{s})
\end{gathered}
$$

